\title{
FLOW AND STABILITY OF A SECOND GRADE FLUID BETWEEN TWO PARALLEL PLATES ROTATING ABOUT NONCOINCIDENT AXES
}

\author{
K. R. RAJAGOPAL \\ Department of Mechanical Engineering, The Catholic University of America, Washington, DC 20064, \\ U.S.A. \\ and
}

A. S. GUPTA

Department of Mechanical Engineering and Applied Mechanics, The University of Michigan, Ann Arbor, MI 48109, U.S.A.

(Communicated by T. W. TING)

\begin{abstract}
An exact solution is given for the flow of a second grade fluid between two infinite parallel plates rotating about noncoincident axes normal to the plates. A comparison of the torque and force exerted by the fluid on the plates with the corresponding experimental values would enable one to determine the normal stress moduli. Using energy method, the stability of the above flow is investigated for disturbances of finite amplitude. It is found that the region of sure stability decreases with increase in the viscoelastic parameter
\end{abstract}

\section{INTRODUCTION}

THE ORTHOGONAL rheometer of Maxwell and Chartoff [1] is used for determining the complex dynamic viscosity of a viscoelastic fluid. It consists of two parallel plates which rotate with the same constant angular velocity about two parallel noncoincident axes. Abbot and Walters [2] gave an exact solution for the flow of an incompressible viscous fluid between two infinite parallel plates which rotate with the same angular velocity about two nonconincident axes. They also studied the flow of a viscoelastic fluid (obeying the model due to Walters [3]) in the same domain assuming that the distance between the axes of rotation is small. The flows investigated in Ref.[2] have relevance to the flows generated in a Maxwell-Chartoff rheometer mentioned above, the plates being, of course, of finite dimension. However, the analysis in [2] for a viscoelastic fluid employs an expansion of the appropriate physical variables in terms of a power series in the distance between the axes of rotation essentially ignoring terms of quadratic and higher order in the series.

In this paper we establish an exact solution for the flow of an incompressible second grade fluid between two infinite parallel plates rotating with the same constant angular velocity about two noncoincident axes normal to the plates. The investigations of Berker [4] and Rajagopal and Gupta [5] who studied the flow of an incompressible viscous fluid and an incompressible second grade fluid, respectively, between two infinite parallel plates rotating with a constant angular velocity about a common axis of rotation having bearing on the problem studied in this paper.

It is worth observing that the equations of motion of an incompressible second grade fluid are in general of higher order than the Navier-Stokes equations. Thus obtaining an exact solution to these equations might, in general, require boundary conditions in addition to the usual no slip conditions. Further the nonlinearities which occur in the equations of motion of a second grade fluid are of higher order than those in the Navier-Stokes equations. In this context we refer the reader to $[5,6]$ for further discussion. It is also appropriate to remark that a general class of non steady flows in a second grade fluid, namely the flow between two infinite parallel planes due to constant tangential surface forces and the flows through channels and pipes under constant pressure gradient with the assumption of constant dynamic viscosity and positive normal stress modulus, was investigated by Ting [7].

It turns out that the form of the solution for the velocity field in this paper is similar to that in [2] although the pressure fields in these problems differ. It is also worth emphasizing that unlike [2] our solution does not require that the distance between the axes of rotation be small, the results being valid for arbitrary distances between the axes. The solution established in [5] 
for coincident axes, however, cannot be obtained as a limiting case of the solution obtained here for noncoincident axes.

Using the energy method[8], we also study the stability of the flow in this problem for disturbances of finite amplitude. Here we extend the general stability analysis of Dunn and Fosdick [9] for flows of a second grade fluid to unbounded domains. We examine the stability of the base flow in terms of two nondimensional numbers, viz. $R$ (the Reynolds number based on the angular velocity of rotation and the distance between the plates) and a viscoelastic parameter $\Gamma$ (denoting the ratio of elastic to viscous forces). As in [5], it is found that as $\Gamma$ increases, the domain of sure stability decreases. The uniqueness of the solution for the equations of motion of a second grade fluid depends on $\Gamma[10]$.

In Section 2 we provide a mathematical formulation of the present boundary value problem. The main result concerning the exact solution for velocity and pressure distribution in the flow is given in Section 3. Finally in Section 4 we study the stability of this flow.

\section{MATHEMATICAL FORMULATION}

The stress $T$ in a homogeneous Rivlin-Ericksen fluid of second grade is given by ([11])

$$
\mathbf{T}=-p \mathbf{I}+\mu \mathbf{A}_{1}+\alpha_{1} \mathbf{A}_{2}+\alpha_{2} \mathbf{A}_{1}^{2}
$$

where $\mu$ is the dynamic viscosity coefficient, $\alpha_{1}$ and $\alpha_{2}$ are the normal stress moduli. We shall assume that the material moduli are constants independent of temperature. Further $-p \mathbf{I}$ denotes the constitutively indeterminate part of the stress due to the assumption of incompressibility and $\mathbf{A}_{1}$ and $\mathbf{A}_{2}$ stand for the first two Rivlin-Ericksen tensors defined through

$$
\mathbf{A}_{1}=\operatorname{grad} \mathbf{v}+(\operatorname{grad} \mathbf{v})^{T}
$$

and

$$
\mathbf{A}_{2}=\dot{\mathbf{A}}_{1}+(\operatorname{grad} \mathbf{v})^{T} \mathbf{A}_{1}+\mathbf{A}_{1}(\operatorname{grad} \mathbf{v})
$$

where $\mathbf{v}$ is the velocity field and the overdot denotes material time differentiation.

In this analysis we shall consider the model represented by (2.1) as an exact model. This model was shown to be a second order approximation to the response functional of a simple fluid in the sense of retardation by Coleman and Noll[12]. But since the model is properly frame-invariant it can also be considered as an exact model in its own right. However, the two points of view are not contradictory and in this context we refer the reader to $[9,13]$ for further comments. If the model (2.1) is required to be compatible with thermodynamics in the sense that all motions satisfy the Clausius-Duhem inequality and the assumption that the specific Helmholtz free energy is a minimum in equilibrium (at constant temperature), then the material moduli must meet the following restrictions [9])

$$
\mu \geq 0, \quad \alpha_{1} \geq 0 \text { and } \alpha_{1}+\alpha_{2}=0 .
$$

The third condition in (2.4) is a consequence of the Clausius-Duhem inequality while the second follows from the requirement that the specific Helmholtz free energy is a minimum in equilibrium. More recently the implications of requiring the specific internal energy rather than the specific Helmholtz free energy be a minimum was investigated in [14].

When (2.1) is substituted into the equation of linear momentum

$$
\operatorname{div} \mathbf{T}+\rho \mathbf{b}=\rho \dot{\mathbf{v}}
$$

where $\mathbf{b}$ denotes the body force per unit mass, one obtains

$$
\mu \Delta \mathbf{v}+\alpha_{\mathrm{l}}(\Delta \omega \times \mathbf{v})+\alpha_{\mathrm{t}} \Delta \mathbf{v}_{\mathrm{t}}+\rho \mathbf{v}_{\mathrm{t}}-\rho(\boldsymbol{\omega} \times \mathbf{v})=\operatorname{grad} \hat{P}
$$


where $\omega=$ curl $\mathbf{v}$ and

$$
\hat{P}=p-\alpha_{1}\left[(\mathbf{v} \cdot \Delta \mathbf{v})+\frac{1}{4}\left|\mathbf{A}_{1}\right|^{2}\right]+\rho \phi+\frac{1}{2} \rho|\mathbf{v}|^{2} .
$$

Here we have assumed that $b$ is conservative so that $b=-\operatorname{grad} \phi$ for some scalar field $\phi$. Also since the fluid is incompressible it can undergo only isochoric motions and hence

$$
\operatorname{div} \mathbf{v}=\mathbf{0}
$$

In (2.6) $\left|\mathbf{A}_{1}\right|$ and $|\mathbf{v}|$ denote the usual trace norm $\left|\mathbf{A}_{1}\right|^{2}=\operatorname{tr} \mathbf{A}_{1} \mathbf{A}_{1}^{T}$ and $(\mathbf{v} \cdot \mathbf{v})^{1 / 2}$, respectively. Further in (2.5) the subscript $t$ denotes partial derivative with respect to time.

We conclude this section with a formal statement of the boundary value problem. We wish to determine the distribution of velocity and pressure satisfying (2.5) and (2.7) for the problem of steady flow between two infinite parallel plates rotating with constant angular velocity $\Omega$ about two noncoincident axes normal to the plates, the distance between the axes of rotation being $a$. A Cartesian coordinate system $O x y z$ is introduced so that the upper and lower plates are defined by $z=h$ and $z=0$, respectively. The upper plate rotates about an axis through $P$ and the lower plate rotates about an axis through $Q$ as shown in Fig. 1.

The appropriate boundary conditions for the velocity field are

$$
u=\frac{\Omega a}{2}-\Omega y, \quad v=\Omega x, \quad w=0 \quad \text { at } \quad z=h
$$

and

$$
u=-\frac{\Omega a}{2}-\Omega y, \quad v=\Omega x, \quad w=0 \quad \text { at } \quad z=0
$$

where $u, v$ and $w$ denote the velocity components along the $x, y$ and $z$ directions.

We seek steady solutions of the form

$$
u=-\Omega(y-g(z)), \quad v=\Omega(x-f(z)), \quad w \equiv 0
$$

which satisfy (2.7).

To determine the velocity field we shall find it convenient to eliminate pressure by taking

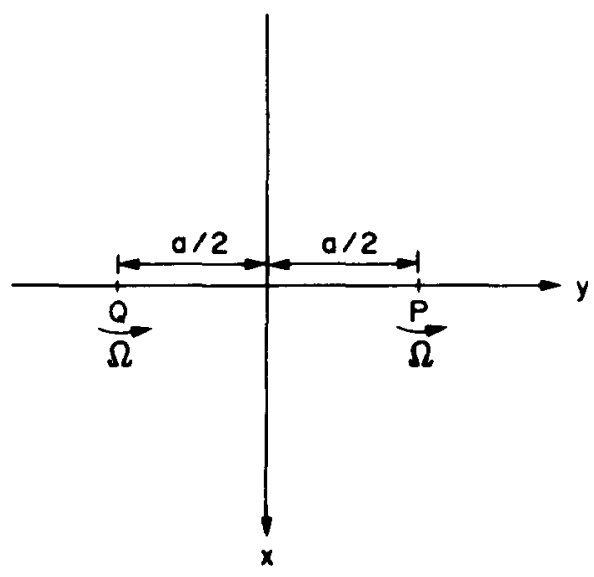

Fig. 1. 
curl of (2.5) as follows

$$
\mu \Delta \omega+\alpha_{1} \operatorname{curl}(\Delta \omega x v)+\alpha_{1} \Delta \omega_{t}-\rho \operatorname{curl}(\omega x v)=0
$$

which constitutes the starting point of our analysis.

\section{EXACT SOLUTION}

On substituting (2.10) in (2.11) we obtain

$$
\mu f^{\prime \prime \prime}+\alpha_{1} \Omega g^{\prime \prime \prime}+\rho \Omega g^{\prime}=0
$$

and

$$
\mu g^{\prime \prime \prime}-\alpha_{1} \Omega f^{\prime \prime \prime}-\rho \Omega f^{\prime}=0
$$

where a prime denotes differentiation with respect to $z$. It follows from $(2.8)-(2.10)$ that

$$
f(0)=0, f(h)=0, \quad g(0)=-\frac{a}{2}, g(h)=\frac{a}{2}
$$

Let us introduce $F(z)=f+i g$ where $i=(-1)^{1 / 2}$. Equations (3.1) and (3.2) can clearly be combined as

$$
F^{\prime \prime \prime}-(m+i n)^{2} F^{\prime}=0
$$

where $m$ and $n$ are given by

$$
m^{2}=\frac{\rho\left\{\left[(\mu / \Omega)^{2}+\alpha_{1}^{2}\right]^{1 / 2}-\alpha_{1}\right\}}{2\left[(\mu / \Omega)^{2}+\alpha_{1}^{2}\right]}
$$

and

$$
n^{2}=\frac{\rho\left\{\left[(\mu / \Omega)^{2}+\alpha_{1}^{2}\right]^{1 / 2}+\alpha_{1}\right\}}{2\left[(\mu / \Omega)^{2}+\alpha_{1}^{2}\right]}
$$

Now from (2.5) and (2.10), we have

$$
\begin{aligned}
& \frac{1}{\rho} \frac{\partial P}{\partial x}=\Omega^{2}(x-f)+\nu \Omega g^{\prime \prime} \\
& \frac{1}{\rho} \frac{\partial P}{\partial y}=\Omega^{2}(y-g)-\nu \Omega f^{\prime \prime} \\
& \frac{1}{\rho} \frac{\partial P}{\partial z}=\frac{\alpha_{1} \Omega^{2}}{\rho}\left[(x-f) f^{\prime \prime \prime}+(y-g) g^{\prime \prime \prime}\right]
\end{aligned}
$$

where

$$
P=\hat{P}-\frac{1}{2} \rho|\mathbf{v}|^{2}
$$

Integration of (3.1) and (3.2) gives

$$
\begin{array}{r}
\mu f^{\prime \prime}+\alpha_{1} \Omega g^{\prime \prime \prime}+\rho \Omega g=q \\
\mu g^{\prime \prime}-\alpha_{1} \Omega f^{\prime \prime}-\rho \Omega f=s
\end{array}
$$

where $q$ and $s$ are constants. 
Substituting (3.7)-(3.9) in

$$
\mathrm{d} P / \rho=[(\partial P / \partial x) \mathrm{d} x+(\partial P / \partial y) \mathrm{d} y+(\partial P / \partial z) \mathrm{d} z] / \rho
$$

and integrating the resulting equation, we obtain after using (3.11) and (3.12) the following expression for the pressure

$$
\frac{P}{\rho}=\frac{\Omega}{2}\left(x^{2}+y^{2}\right)+\frac{\alpha_{1} \Omega^{2}}{\rho}\left[2 x f^{\prime \prime}+2 y g^{\prime \prime}-f^{\prime \prime}-g g^{\prime \prime}+1 / 2\left(f^{\prime 2}+g^{\prime 2}\right)\right]+\left(\frac{s x}{\rho}-\frac{q y}{\rho}\right)+C
$$

where $C$ is an arbitrary constant. It can be seen from (3.13) that nonzero values of $s$ and $q$ would give rise to a pressure gradient between the plates with a corresponding flow of Poiseuille type. In order to remove the possibility of flow of this type and at the same time to ensure the symmetry of the velocity distribution about the plane $z=h / 2$, we put $s=q=0[2]$. Equations (3.11) and (3.12) now give upon using (3.3)

$$
f^{\prime \prime}(0)=\frac{\nu \Omega a / 2}{\left[\nu^{2}+\left(\alpha_{1} \Omega / \rho\right)^{2}\right]}, \quad g^{\prime \prime}(0)=\frac{\alpha_{1} \Omega^{2} a / 2 \rho}{\left[\nu^{2}+\left(\alpha_{1} \Omega / \rho\right)^{2}\right]}
$$

which yield

$$
F^{\prime \prime}(0)=\frac{(\nu \Omega a / 2)+i\left(\alpha_{1} \Omega^{2} a / 2 \rho\right)}{\left[\nu^{2}+\left(\alpha_{1} \Omega / \rho\right)^{2}\right]}
$$

Since (3.3) gives

$$
F(0)=-i a / 2, \quad F(h)=i a / 2
$$

the solution of (3.4) satisfying (3.14) and (3.15) is

$$
F(z)=\frac{i a}{2}\left[\frac{\mathrm{e}^{(m+i n) z}-\mathrm{e}^{-(m+i n) z}+\mathrm{e}^{(m+i n)(z-h)}-\mathrm{e}^{-(m+i n)(z-h)}}{\mathrm{e}^{(m+i n) h}-\mathrm{e}^{-(m+i n) h}}\right] .
$$

This gives after a lengthy but straightforward computation

$$
\begin{aligned}
f(z)= & \frac{2 a}{\Delta}\{\sin n h \cosh m h[\cos n z \sinh m z+\cos n(z-h) \sinh m(z-h)] \\
& -\cos n h \sinh m h[\sin n z \cosh m z+\sin n(z-h) \cosh m(z-h)\}
\end{aligned}
$$

and

$$
\begin{aligned}
g(z)= & \frac{2 a}{\Delta}\{\cos n h \sinh m h[\cos n z \sinh m z+\cos n(z-h) \sinh m(z-h)] \\
& +\sin n h \cosh m h[\sin n z \cosh m z+\sin n(z-h) \cosh m(z-h)]\}
\end{aligned}
$$

where

$$
\Delta=4\left(\sinh ^{2} m h+\sin ^{2} n h\right) .
$$

Thus the velocity and pressure fields are obtained from (2.10) and (3.13) with $q=s=0$ and $f$ and $g$ given by (3.17) and (3.18). The expressions for the velocity components in (2.10) agree with those in [2] when $\alpha_{1}=0$, i.e. in the case of the Navier-Stokes equations. Of course, as is to be expected, both the velocity and pressure fields depend on the normal stress modulus $\alpha_{1}$. Although the velocity field in the present problem has the same form as that in [2], the pressure fields in the two problems are markedly different. 
As mentioned earlier, the orthogonal rheometer of Maxwell and Chartoff [1] can be used in determining the complex dynamic viscosity of a second grade fluid. The velocity obtained above may be used to determine the stress at any point and hence the torque and the normal force exerted by the fluid on the two plates. This can be compared with experimentally measured torque and normal forces in the orthogonal rheometer which would enable one to determine the normal stress moduli.

\section{STABILITY}

In this section we study the stability of the above flow subject to disturbances of finite amplitude. Let $v$ and $\hat{P}$ denote the velocity and pressure (modified) for the base flow which satisfy (2.5) and (2.7). Further assume that $v^{\prime}$ and $\hat{P}^{\prime}$ denote another distribution of velocity and pressure (modified) which satisfy the same equations and boundary conditions as $v$ and $P$ but may conform to different initial conditions. Let

$$
\mathbf{u}=\mathbf{v}^{\prime}-\mathbf{v} \text { and } \hat{p}=\hat{P}^{\prime}-\hat{P}
$$

denote the difference fields. Sufficient conditions for the disturbance field to decay asymptotically to the mull flow were obtained by Dunn and Fosdick [9] using the energy method [8]. They showed that for a bounded domain $B$, the disturbance velocity decays asymptotically to zero in the sense that

$$
\begin{gathered}
\int_{B}|\mathbf{u}(\mathbf{x}, t)|^{2} \mathrm{~d} V+\frac{\alpha_{1}}{\rho} \int_{B} \mid \operatorname{grad} \mathbf{u}(\mathbf{x}, t)^{2} \mathrm{~d} V \\
\leq \mathrm{e}^{-\lambda t}\left[\int_{B}|\mathbf{u}(\mathbf{x}, 0)|^{2} \mathrm{~d} V+\frac{\alpha_{1}}{\rho} \int_{B}|\operatorname{grad} \mathbf{u}(\mathbf{x}, 0)|^{2} \mathrm{~d} V\right]
\end{gathered}
$$

where $\lambda>0$, provided the material moduli and the base flow are such that the following inequality holds

$$
\frac{2 \alpha_{1}}{\rho}(M+\bar{M})+K\left(\frac{\alpha_{1} N}{\rho}+\bar{M}\right)-\frac{2 \mu}{\rho}<0
$$

The expression on the 1.h.s. of (4.2) is a measure of the kinetic energy and the energy due to stretching in the fluid. In (4.3), $M$ and $-\bar{M}$ denote the maximum and minimum of the eigenvalues of the Rivlin-Ericksen tensor $\mathbf{A}_{1}, N$ denotes the maximum eigenvalue of $\Delta \mathbf{A}_{1}$ and $K$ stands for the Poincare coefficient for the domain. Since the domain is unbounded in the present problem, we make the following assumptions regarding the asymptotic behaviour of $\mathbf{u}$ and $\hat{p}$ [8]

$$
u=0\left(r^{-k}\right), \operatorname{grad} u=0\left(r^{-k-1}\right) \text { and } \hat{p}=0\left(r^{-k+1}\right)
$$

where $k>1$. These conditions will ensure that appropriate surface integrals will vanish in the limit $r \rightarrow \infty$. It then follows [8] that (4.3) can be replaced by

$$
\frac{2 \alpha_{1}}{\rho}(M+\bar{M})+\frac{h^{2}}{\pi^{2}}\left(\frac{\alpha_{1} N}{\rho}+\bar{M}\right)-\frac{2 \mu}{\rho}<0 .
$$

We shall study (4.5) in detail. It follows from (2.2) and (2.10) that the eigenvalues of $\mathbf{A}_{1}$ are

$$
\lambda=0 \quad \text { and } \quad \lambda= \pm \Omega\left\{\left(g^{\prime}\right)^{2}+\left(f^{\prime}\right)^{2}\right\}^{1 / 2}
$$

and the eigenvalues of $\Delta \mathbf{A}_{1}$ are

$$
\Lambda=0 \quad \text { and } \quad \Lambda= \pm \Omega\left\{\left(g^{\prime \prime \prime}\right)^{2}+\left(f^{\prime \prime \prime}\right)^{2}\right\}^{1 / 2} .
$$


It now follows from (4.6) and (4.7) that

$$
M=\bar{M} \geqslant \Omega\left\{\left(g^{\prime}\right)^{2}+\left(f^{\prime}\right)^{2}\right\}^{1 / 2} \text { for } 0 \leq z \leq h
$$

and

$N$

$$
N \geqslant \Omega\left\{\left(g^{\prime \prime \prime}\right)^{2}+\left(f^{\prime \prime \prime}\right)^{2}\right\}^{1 / 2} \text { for } 0 \leq z \leq h .
$$

Substitution of $f$ and $g$ from (3.17) and (3.18) into (4.8) and (4.9) gives

$$
\begin{aligned}
M= & \bar{M} \geqslant \Omega\left[\left(m K_{1}-n S\right)^{2}+\left(m S+n K_{1}\right)^{2}\right]^{1 / 2}\left\{[\psi(z)+\psi(z-h)]^{2}\right. \\
& \left.+[\phi(z)+\phi(z-h)]^{2}\right\}^{1 / 2}
\end{aligned}
$$

and

$$
\begin{aligned}
N \geqslant & \Omega\left\{\left[n^{3} K_{1}-3 n m^{2} K_{1}+3 m n^{2} S-m^{3} S\right]^{2}+\left[m^{3} K_{1}\right.\right. \\
& \left.\left.-3 m n^{2} K_{1}-3 m^{2} n S+n^{3} S\right]^{2}\right\}^{1 / 2}\left\{[\psi(z)+\psi(z-h)]^{2}\right. \\
& \left.+[\phi(z)+\phi(z-h)]^{2}\right\}^{1 / 2}
\end{aligned}
$$

for $0 \leq z \leq h$ with

$$
\begin{aligned}
K_{1} & =\frac{2 a}{\Delta} \sin n h \cosh m h, S=\frac{2 a}{\Delta} \cos n h \sinh m h \\
\phi(z) & =\sin n z \sinh m z, \quad \psi(z)=\cos n z \cosh m z .
\end{aligned}
$$

Now a straightforward computation yields from (4.13)

$$
\begin{gathered}
{[\psi(z)+\psi(z-h)]^{2}+[\phi(z)+\phi(z-h)]^{2}} \\
=\cosh ^{2} m z-\sin ^{2} n z+\cosh ^{2} m(h-z)-\sin ^{2} n(h-z)
\end{gathered}
$$

$+2 \sin n z \sinh m z \sin n(h-z) \sinh m(h-z)+2 \cos n z \cosh m z \cos n(z-h) \cosh m(h-z)$.

It follows from (3.5) and (3.6) that $n \geq m$ and hence for $0 \leq z \leq h$,

$$
\cosh ^{2} m z-\sin ^{2} n z \leq \cosh ^{2} n z-\sin ^{2} n z \leq \cosh ^{2} n h-\sin ^{2} n h .
$$

Similarly

$$
\cosh ^{2} m(h-z)-\sin ^{2} n(h-z) \leq \cosh ^{2} n h-\sin ^{2} n h .
$$

Further

$$
2 \sin n z \sinh m z \sin n(h-z) \sinh m(h-z) \leq 2 \sinh ^{2} m h
$$

and

$$
2 \cos n z \cosh m z \cos n(z-h) \cosh m(h-z) \leq 2 \cosh ^{2} m h .
$$

Now from $(4.14)-(4.18)$ we have

$$
[\psi(z)+\psi(z-h)]^{2}+[\phi(z)+\phi(z-h)]^{2} \leq 2\left(\cosh ^{2} n h-\sin ^{2} n h\right)+2 \cosh 2 m h .
$$

Substituting (4.10) and (4.11) in (4.5) and using (4.12) and (4.19), we finally obtain the 


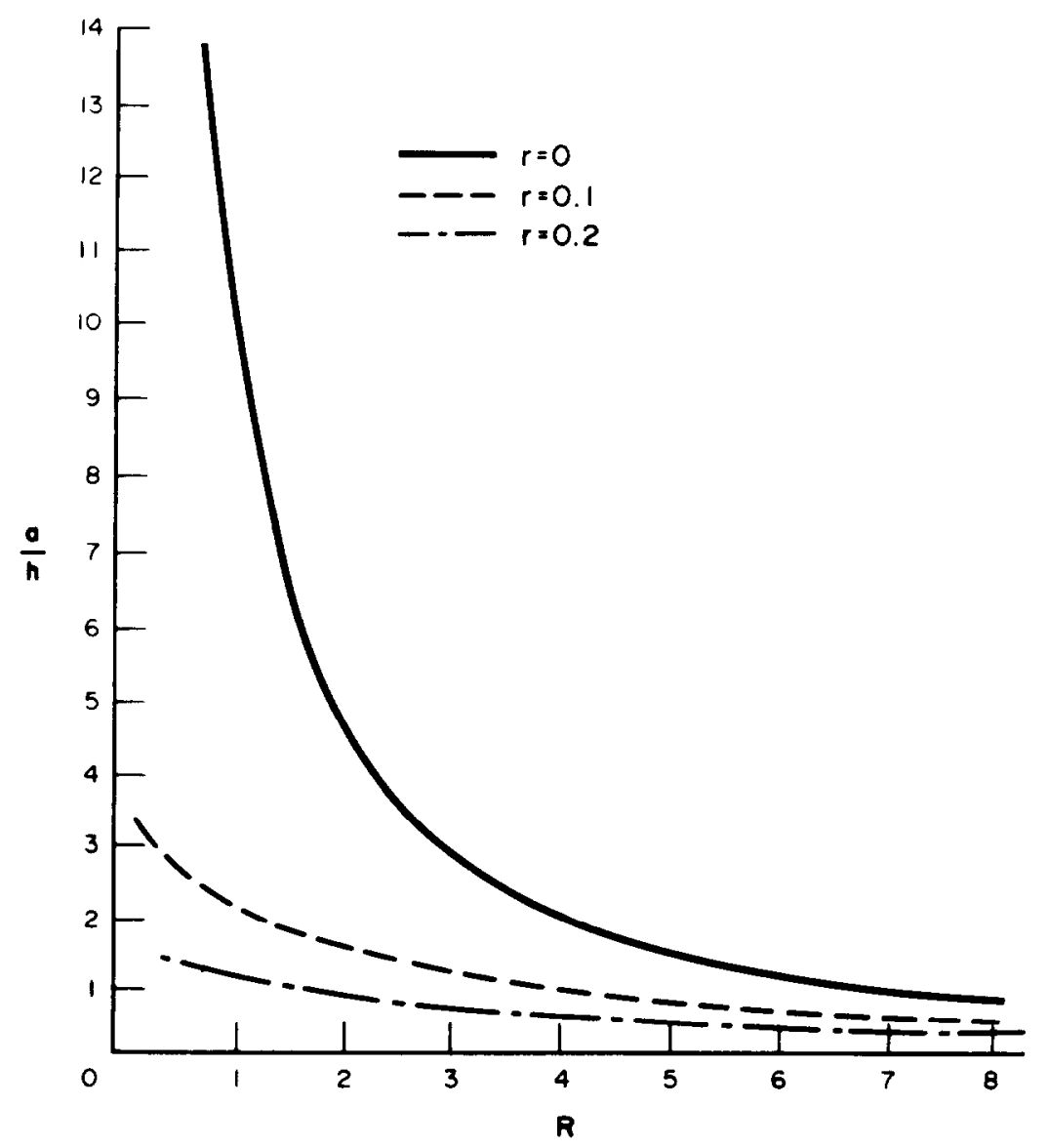

Fig. 2.

sufficient condition for stability of the base flow subject to arbitrary disturbances as $\frac{a}{h}\left\{\left(4 \Gamma+\frac{R}{\pi^{2}}\right)\left[(m h \sin n h \cosh m h-n h \cos n h \sinh m h)^{2}+(m h \cos n h \sinh m h\right.\right.$ $\left.+n h \sin n h \cosh m h)^{2}\right]^{1 / 2}+\frac{\Gamma}{\pi^{2}}\left[\left(n^{3} h^{3} \sin n h \cosh m h-3 m^{2} n h^{3} \sin n h \cosh m h\right.\right.$ $\left.+3 m n^{2} h^{3} \cos n h \sinh m h-m^{3} h^{3} \cos n h \sinh m h\right)^{2}+\left(m^{3} h^{3} \sin n h \cosh m h\right.$ $\left.\left.\left.-3 m n^{2} h^{3} \sin n h \cosh m h-3 m^{3} n h^{3} \cos n h \sinh m h+n^{3} h^{3} \cos n h \sinh m h\right)^{2}\right]^{1 / 2}\right\}$

$$
<\Delta\left[2\left(\cosh ^{2} n h-\sin ^{2} n h\right)+2 \cosh 2 m h\right]^{-1 / 2}
$$

where

$$
R=\frac{h^{2} \Omega}{\nu}, \quad \Gamma=\frac{\alpha_{1} \Omega}{\rho \nu}
$$

Figure 2 displays the stability region in the $(a / h)-R$ plane as computed from (4.20) for several values of the viscoelastic paramerer $\Gamma$. The region bounded by a curve (for fixed $\Gamma$ ) and the coordinate axes represents the domain of sure stability with respect to finite amplitude disturbances. It may be noticed that this domain of sure stability decreases with increase in $\Gamma$. This tends to suggest that viscoelasticity could possibly exert a destabilizing influence on the flow. The region outside the domain of sure stability is not necessarily a region of instability since the above estimate is based on a sufficient condition for stability. 


\section{REFERENCES}

[1] B. MAXWELL and R. P. CHARTOFF; Trans. Soc. Rheol. 9, 41 (1965).

[2] T. N. G. ABBOTT and K. WALTERS, J. Fluid Mech. 40, 205 (1970).

[3] K. WALTERS, J. Fluid Mech. 40, 191 (1970).

[4] R. BERKER, Arch. Mech. Stos. 31, 265 (1979).

[5] K. R. RAJAGOPAL and A. S. GUPTA, Flow and stability of second grade fluids between two parallel rotating plates. Arch. Mech. Stos. (1981).

[6] K. R. RAJAGOPAL and A. S. GUPTA, Intl. J. Engng Sci, 19, 1009 (1981).

[7] T. W. TING, Arch. Rat. Mech. Anal. 14, 1 (1963).

[8] J. SERRIN, Handbuch der Physik, Vol. VIII/1, p. 125, Berlin (1959).

[9] J. E. DUNN and R. L. FOSDICK, Arch. Rat. Mech. Anal. 56, 191 (1974).

[10] R. L. FOSDICK and K. R. RAJAGOPAL, Int. J. Nonlinear Mech. 13, 131 (1978).

[11] R. S. RIVLIN and J. L. ERICKSEN, J. Rat. Mech. Anal. 4, 323 (1955).

[12] B. D. COLEMAN and W. NOLL, Arch. Rat. Mech. Anal. 6, 355 (1960).

[13] R. L. FOSDICK and K. R. RAJAGOPAL, Arch. Rat. Mech. Anal. 70145 (1970).

[14] K. R. RAJAGOPAL and A. S. WINEMAN, Int. J. Engng Sci. In press.

(Received 23 January 1981) 